\title{
Expression of chosen cell cycle and proliferation markers in pancreatic intraepithelial neoplasia
}

\author{
Justyna Zińczuk ${ }^{1}$, Konrad Zaręba ${ }^{2}$, Katarzyna Guzińska-Ustymowicz ${ }^{1}$, Bogusław Kędra², Andrzej Kemona ${ }^{1}$, \\ Anna Pryczynicz ${ }^{1}$ \\ ${ }^{1}$ Department of General Pathomorphology, Medical University of Bialystok, Bialystok, Poland \\ ${ }^{2} 2^{\text {nd }}$ Clinical Department of General and Gastroenterological Surgery, Medical University of Bialystok, Bialystok, Poland
}

Gastroenterology Rev 2018; 13 (2): 118-126

DOI: https://doi.org/10.5114/pg.2018.75824

Key words: PanIN, pancreatic cancer, cell cycle, proliferation.

Address for correspondence: Justyna Zińczuk, Department of General Pathomorphology, Medical University of Bialystok,

13 Waszyngtona St, 15-269 Bialystok, Poland, phone: +48 663480 618, e-mail: j.zinczuk@wp.pl

\begin{abstract}
Introduction: Pancreatic ductal adenocarcinoma is one of the most aggressive tumours that develops from precursor lesions, most frequently including pancreatic intraepithelial neoplasia (PanIN). Deregulation of the cell cycle, responsible for uncontrolled cell proliferation, is an important phenomenon in the development of this cancer.

Aim: To evaluate the cell cycle and the expression of proliferation markers, namely Ki67, PCNA, and cyclin D1 in pancreatic intraepithelial neoplasia at its different stages of progression.

Material and methods: The study group consisted of 70 patients with different pancreatic diseases (pancreatic ductal adenocarcinoma, pancreatitis, and pancreatic cysts), who also had pancreatic intraepithelial neoplasia. Expression of Ki67, PCNA, and Cyclin D1 was analysed immunohistochemically using appropriate antibodies.

Results: Statistically significant differences were demonstrated in Ki67, PCNA, and Cyclin D1 expression between normal pancreatic ducts and various stages of PanIN $(p<0.001)$. Expression of these proteins increased from normal pancreas to PanIN 1,2 , and 3. Expression of these proteins was higher in stages PanIN 1, 2, and 3 compared to normal pancreas. The expression of Ki67, PCNA, and cyclin D1 was associated with age $(p<0.001)$, Ki67 and PCNA with sex $(p<0.001)$, and PCNA with the type of primary disease $(p=0.031)$. Simultaneously, a directly proportional relationship was established between the expression of all proteins examined $(p<0.001)$.

Conclusions: An increase in the expression of Ki67, PCNA, and cyclin D1 suggests that these proteins may enhance epithelial cell proliferation and may influence the development of pancreatic intraepithelial neoplasia. Moreover, immunohistochemical assessment of Ki67, PCNA, and cyclin D1 expression may be helpful in the differential diagnosis of PanIN.
\end{abstract}

\section{Introduction}

Pancreatic ductal adenocarcinoma (PDAC) is one of the most common neoplasias as well as the most deadly cancer of the highest mortality, with a 5 -year survival rate not exceeding $5 \%$. This is connected with late diagnosis of this cancer resulting in only $15 \%$ of patients undergoing surgical resection. According to the latest reports of the American Cancer Society (ACS), resection of a tumour of size not exceeding $2 \mathrm{~cm}$ increases the 5 -year survival rate by $50 \%$, while for the lesions with diameter less than $1 \mathrm{~cm}$, this rate equals nearly $100 \%$, which underlines the significance of early detection of this cancer at its early stage. According to the ACS, the morbidity of pancreatic cancer will equal around 53,670 and the mortality, about 43,090 in 2017 in the USA [1]. Thus, it is vital to study the biology of pancreatic ductal adenocarcinoma because it may contribute to better diagnostics and development of new, more efficient therapeutic methods [2]. Formulating the model of gradual progression of pancreatic cancer and proving that it develops from the precursor lesions, including the most frequent intraepithelial neoplasia of the pancreas, have become a significant step in the research into pancreatic cancer. In 1999, during the meeting of the Pancreatic Cancer Think Thank (Utah, USA) this international group of pathologists established new nomenclature and uniform histological criteria for the classification of microscopic precancerous lesions in the pancreatic ducts. In this classification, a degree of 
advancement of architectural and cytological lesions in the ducts was taken into consideration, and PanIN was divided into three categories: PanIN 1 A and 1 B (lowgrade lesions), PanIN 2 (medium-grade lesions), and PanIN 3 (high-grade lesions). In their studies, numerous authors confirmed that many mutations of suppressive genes, among others, TP53, CDKN2A, BRCA2, or DPC4, which control cellular proliferation and prevent neoplasia in normal conditions, mutate in the intraepithelial neoplasia, similarly to cancer [3]. Disordered functioning of a cell process is usually caused by the dysfunction of the proteins taking part in the regulation of this process, resulting in uncontrolled proliferation of cells with the active participation of Ki67 protein, Cyclin D1, or PCNA protein. Ki67 is a determinant of proliferation, i.e. fractions of cells multiplying in a given population, and can be used in routine histopathological diagnostics of cancers. The stage of this protein expression is used in the diagnostics and classification of lymphomas, diagnostics of melanoma, dysplasia in the course of ulcerative colitis, or in differential diagnostics of basal cell carcinoma of prostate and basal cell hyperplasia [4]. Expression of Ki67 protein has been used not only in diagnostics of invasive lesions but in precursor lesions, among others, in intraepithelial neoplasia of the cervix as well as in precancerous lesions of the head and neck [5]. Cyclin D1 is another protein with wide diagnostic application, which takes part in the regulation of the cell cycle. This protein is coded by CCND 1 gen (PRAD 1), located on chromosome 11q13, included in the group of proto-oncogenes. It belongs to the family of cyclins regulating the $\mathrm{G} 1$ phase of the cell cycle and is responsible for Protein-Serine-Threonine Kinases of CDK4 and CDK6, with which it forms complexes necessary to initiate DNA replication in the $S$ phase. During the cellular cycle a level of cyclin increases at the beginning of $\mathrm{G} 1$ phase until crossing a control point at the border of $\mathrm{G} 1 / \mathrm{S}$ phases, after which it starts decreasing significantly. This is caused by the fact that degradation of cyclin is essential to initiate replication because its overexpression precludes passing to the $S$ phase. It has been proven that a direct binding of Cyclin D1 to PCNA inhibits DNA synthesis [6]. In the cell cycle, the level of PCNA protein increases in the final stage of the $G 1$ phase, peaks in the $S$ phase, falls during the $G 2$ phase, and disappears completely during mitosis and in the cells in the G0 phase (a resting phase). Thus, Cyclin D1, similarly to Ki67, is regarded as an indicator of proliferation [7]. Although PCNA is the protein participating in DNA replication, its presence was reported in the cytoplasm and extracellular matrix. There PCNA functions as a factor regulating processes of apoptosis and glycolysis and may work as a ligand of superficial receptors controlling natural cell killer activation [8].

\section{Aim}

Therefore, the aim of our study was to evaluate immunohistochemically Ki67, PCNA, and cyclin D1 expression and assessment of correlation of expressions of these proteins with chosen clinicopathological parameters in patients diagnosed with pancreatic intraepithelial neoplasia. Moreover, considering the role of these proteins in the cell cycle, the objective of the current study was to determine whether there was a correlation between Ki67, PCNA, and cyclin D1.

\section{Material and methods}

\section{Patients}

The study group consisted of 70 patients with different pancreatic diseases (ductal adenocarcinoma, cysts, pancreatitis) operated on in the $2^{\text {nd }}$ Clinical Department of General and Gastroenterological Surgery at the University Hospital in Bialystok, in the years 2006-2014. The characteristics of the study group are shown in Table I. The study was performed in conformity with the Declaration of Helsinki for Human Experimentation and received approval of the Local Bioethics Committee of the Medical University of Bialystok (No. R-I-002/139/2014).

\section{Histopathological examination and identification of ductal lesions}

In brief, formalin-fixed and paraffin-embedded tissue specimens were cut on a microtome into 5- $\mu \mathrm{m}$

Table I. Characteristics of the study group

\begin{tabular}{lc} 
Clinicopathological features & Frequency, $\boldsymbol{n}$ (\%) \\
\hline Sex: & $35(50)$ \\
\hline Male & $35(50)$ \\
\hline Female & $33(47.2)$ \\
\hline Age [years]: & $37(52.8)$ \\
\hline$<60$ & \\
\hline$\geq 60$ & $23(32.9)$ \\
\hline Diagnosis: & $38(54.3)$ \\
\hline Pancreatitis & $9(12.8)$ \\
\hline Pancreatic ductal adenocarcinoma & \\
\hline Pancreatic cysts & $33(47.1)$ \\
\hline Location: & $5(7.1)$ \\
\hline Head & $20(28.6)$ \\
\hline Body & $12(17.2)$ \\
\hline Tail
\end{tabular}


sections and stained with haematoxylin-eosin $(\mathrm{H}+\mathrm{E})$. Routine histopathological analysis included diagnosis of primary disease, but also the presence and stage of pancreatic intraepithelial neoplasia. All slides were reviewed by two independent pathologists for the presence and grade of PanIN lesions in accordance with the guidelines developed by the international group of pathologists at the Pancreas Cancer Think Tank meeting sponsored by the National Cancer Institute and held in Park City, Utah in September 16-19, 1999. Briefly, PanIN $1 \mathrm{~A}$ is an epithelial flat lesion, whereas PanIN 1B is a papillary or micropapillary lesion composed of tall columnar cells with basally located nuclei and abundant supranuclear mucin without cytological atypia. PanIN 2 is a mucinous, epithelial flat or papillary lesion with some nuclear abnormalities including loss of polarity, crowding, enlargement, nuclear stratification, and hyperchromatism. PanIN 3 is usually a papillary or micropapillary architecture with abnormal cribriforming, budding, and luminal necrosis with cytological abnormalities like loss of nuclear polarity, dystrophic goblet cells, atypical mitotic figures, and macronucleoli [7]. The presence of PanINs was evaluated on the slides of the normal pancreatic tissue at least $5 \mathrm{~mm}$ away from the carcinoma, while in the non-neoplastic lesions PanINs were evaluated in the site of an ongoing disease process. In the group of 70 patients, the following lesions were found: normal pancreatic ducts were observed in 35 patients, PanIN $1 \mathrm{~A}$ in 65 patients, PanIN $1 \mathrm{~B}$ in 67 patients, PanIN 2 in 51 patients, and only 21 patients had PanIN 3 (Table II).

\section{Immunohistochemistry}

Tissue blocks were cut using a microtome into 5 - $\mu \mathrm{m}$-thick sections on silanised glasses. The sections were deparaffinised in xylenes and hydrated in alcohols. In order to exhibit an antigen, the tissue sections were heated in a water bath at $99^{\circ} \mathrm{C}$ for $20 \mathrm{~min}$ and then cooled for $20 \mathrm{~min}$ at room temperature in citrate buffer $(\mathrm{pH}=6.0)$. Then they were incubated with
$0.5 \%$ hydrogen peroxide in methanol (Novocastra) to block endogenous peroxidase and, next, with protein block (Novocastra) for 5 min. Incubation with mouse anti-Ki67 antibody (clone MM1, Novocastra, 1 : 200 dilution), rabbit anti-cyclin D1 antibody (clone P2D11F11, Novocastra, 1 : 300 dilution), and rabbit anti-PCNA antibody (clone PC10, Novocastra, $1: 200$ dilution) for $1 \mathrm{~h}$ at room temperature. Following streptavidin-biotin reaction (biotinylated secondary antibody, streptavidin-HRP; Novocastra), the antigen antibody complex was visualised by applying chromogen 3.3'-diaminobenzidine (DAB, Novocastra). Positive and negative controls were performed according to the manufacturer's instructions. The expressions of the proteins were assessed in the pancreatic ductal epithelial cells using the quantitative method. Positively staining nuclei were counted in epithelial cells in normal pancreatic ducts and in pancreatic ducts with various stages of pancreatic intraepithelial neoplasia, and expressed as a percentage.

\section{Statistical analysis}

Statistica 10.0 (Statsoft, Cracow, Poland) was used for statistical analysis. The data were analysed using Spearman's rank correlation test. Correlations between protein expressions depending on PanIN stage were tested with the use of Mann-Whitney's test. A p-value of $<0.05$ was considered statistically significant. Missing data were removed in pairs.

\section{Results}

The positive immunohistochemical reaction of the proteins Ki67, cyclin D1, and PCNA was evaluated in the nucleus of pancreatic ductal epithelial cells (Figures 1 A-D, 2 A-D, 3 A-D). The expression of Ki67 protein ranged from 0 to $50 \%$, while its average value equalled $5.3 \%$. The median was $2 \%$ and the modal value was $5 \%$. The expression of cyclin D1 was in the range 0 to $90 \%$. The mean value of cyclin D1 expression was $20.1 \%$ and the median was $10 \%$, the modal value

Table II. Number of lesions assessed in the group of 70 patients

\begin{tabular}{|c|c|c|c|c|c|c|c|}
\hline Variable & $\begin{array}{c}\text { Normal } \\
\text { pancreatic } \\
\text { ducts }\end{array}$ & PaniN 1a & PanIN 1b & $\begin{array}{c}\text { PanIN } 1 \\
(1 A+1 B)\end{array}$ & PanIN 2 & PanIN 3 & Total (\%) \\
\hline Pancreatitis & 14 (18.2\%) & $21(27.3 \%)$ & $21(27.3 \%)$ & $42(54.6 \%)$ & $16(20.8 \%)$ & $5(6.4 \%)$ & 77 \\
\hline $\begin{array}{l}\text { Pancreatic ductal } \\
\text { adenocarcinoma }\end{array}$ & $14(10.8 \%)$ & 35 (26.9\%) & 37 (28.5\%) & 72 (55.4\%) & 29 (22.3\%) & 15 (11.5\%) & 130 \\
\hline Pancreatic cysts & 7 (21.9\%) & $9(28.1 \%)$ & $9(28.1 \%)$ & $18(56.2 \%)$ & $6(18.8 \%)$ & $1(3.1 \%)$ & 32 \\
\hline $\begin{array}{l}\text { Total number } \\
\text { of lesions }\end{array}$ & 35 (14.7\%) & $65(27.2 \%)$ & 67 (28.0\%) & $132(55.2 \%)$ & $51(21.3 \%)$ & $21(8.8 \%)$ & 239 \\
\hline
\end{tabular}



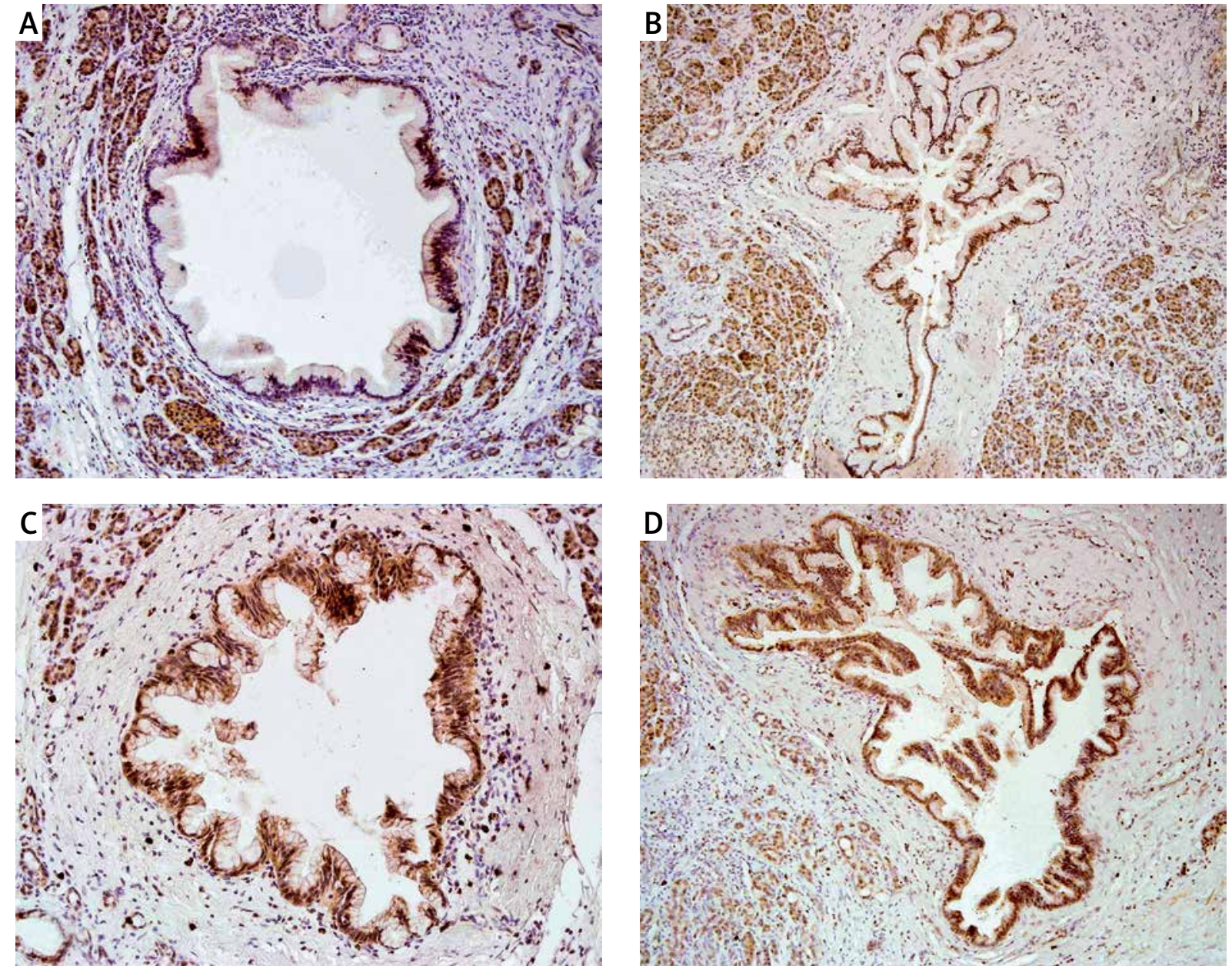

Figure 1. Nuclear immunoreactivity of cyclin D1 in PanIN 1A (A), in PanIN 1B (B), in PanIN 2 (C), and in PanIN 3 (D).

Typical examples are shown

equalled $0 \%$. PCNA expression values were within the range $60-100 \%$, whereas the mean value was $93.9 \%$. The median was $100 \%$ and the mode amounted to $100 \%$.

\section{Ki67, cyclin D1, and PCNA expression in correlation with clinicopathological parameters in pancreatic intraepithelial neoplasia}

Statistical analysis revealed correlations of the Ki67, cyclin D1, and PCNA expressions with the presence and degree of pancreatic intraepithelial neoplasia $(p<0.001)$. Patients above the age of 60 years had increased levels of Ki67, cyclin D1, and PCNA expression $(p<0.001)$. An increase in the expression of Ki67 and PCNA was observed in the male group ( $p=0.001$ and $p$ $<0.001)$. The higher expression of PCNA was shown to be associated with the type of primary disease - pancreatic ductal adenocarcinoma $(p=0.031)$ (Table III).
Comparison of the Ki67, cyclin D1, and PCNA expressions in normal pancreatic ducts and those at different degrees of pancreatic intraepithelial neoplasia

Normal vs. PanIN 1, PanIN 2, and PanIN 3

The expressions of Ki67, cyclin D1, and PCNA proteins were significantly higher in pancreatic intraepithelial neoplasia compared to normal tissue. The mean Ki67 expression was significantly higher in PanIN 1 (4.0\%), PanIN 2 (7.5\%), and PanIN 3 (14.2\%) in comparison with normal pancreatic ducts $(1.2 \%)(p<0.001)$. Similarly, the mean expression of cyclin D1 was significantly higher $(p<0.001)$ in PanIN 1 (12.9\%), PanIN 2 (35.7\%), and PanIN 3 (59.5\%) compared to normal pancreatic ducts $(0.7 \%)$. The mean expression of PCNA was also significantly higher $(p<0.001)$ in PanIN $1(93.8 \%)$, PanIN 2 (96.5\%), and PanIN 3 (97.6\%) compared to normal pancreatic ducts, where the mean protein expression was $88.9 \%$ (Table IV). 

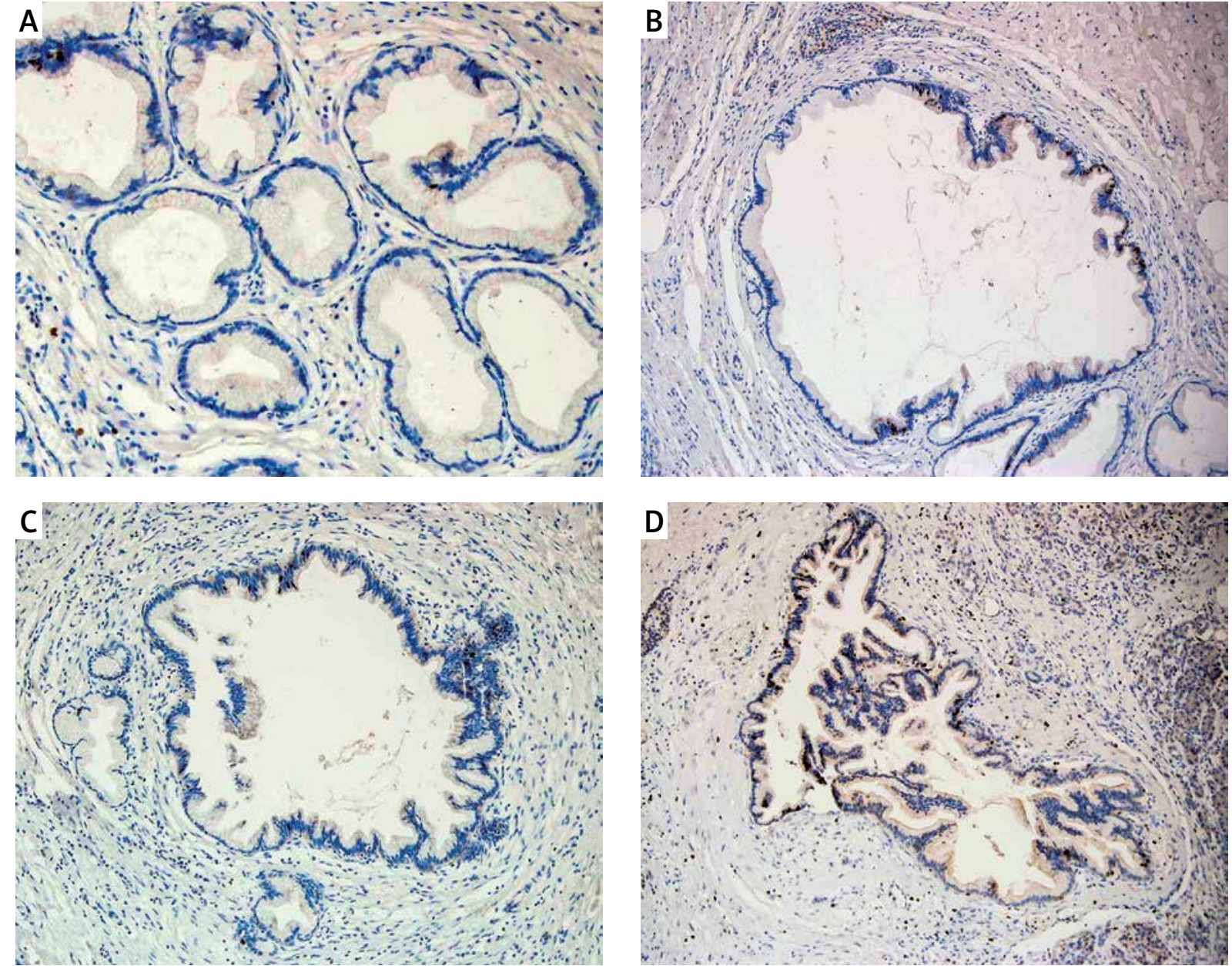

Figure 2. Nuclear immunoreactivity of Ki67 in PanIN 1A (A), in PanIN 1B (B), in PanIN 2 (C), and in PanIN 3 (D). Typical examples are shown

\section{PanIN 1 vs. PanIN 2 and PanIN 3}

The mean expression of Ki67, cyclin D1, and PCNA proteins was significantly higher in PanIN 2 and PanIN 3 in comparison with PanIN 1 ( $p<0.001)$ (Table IV).

\section{Pan IN 2 vs. PanIN 3}

The mean Ki67, cyclin D1, and PCNA proteins protein expression was significantly higher in PanIN 3 compared to PanIN 2 lesions ( $p<0.001)$ (Table IV).

\section{Correlation between expressions of $\mathrm{Ki} 67$, cyclin D1, and PCNA proteins}

Statistical analysis showed a statistically significant correlation between expressions of Ki67, cyclin D1, and PCNA proteins. This relationship was directly proportional, which means that in the case of an increased expression of Ki67 protein the increased expression of other proteins was observed (Figures 4-6).

\section{Discussion}

Uncontrolled proliferation is one of characteristic features of neoplasm. One of the indexes of cell proliferation is Ki67 protein present in phases $G 1, S, G 2$, and $M$ of the cell cycle, and it is found in multiplying cells, both normal and cancerous. In immunohistochemistry, Ki67 is detected by application of MIB-1 antibody showing a positive expression in most cases of ductal pancreatic adenocarcinoma [9]. Despite the common use of Ki67 antibody in histopathological diagnostics of many cancers, there are few studies describing the expression and significance of this protein in the development of precancerous lesions in pancreas. In our study, Ki67 expression was assessed and a statistically significant correlation was proven between this protein expression and the presence and staging of intraepithelial neoplasia in the pancreas. Ki67 expression was observed to be enhanced with the increasing staging of intraepithe- 

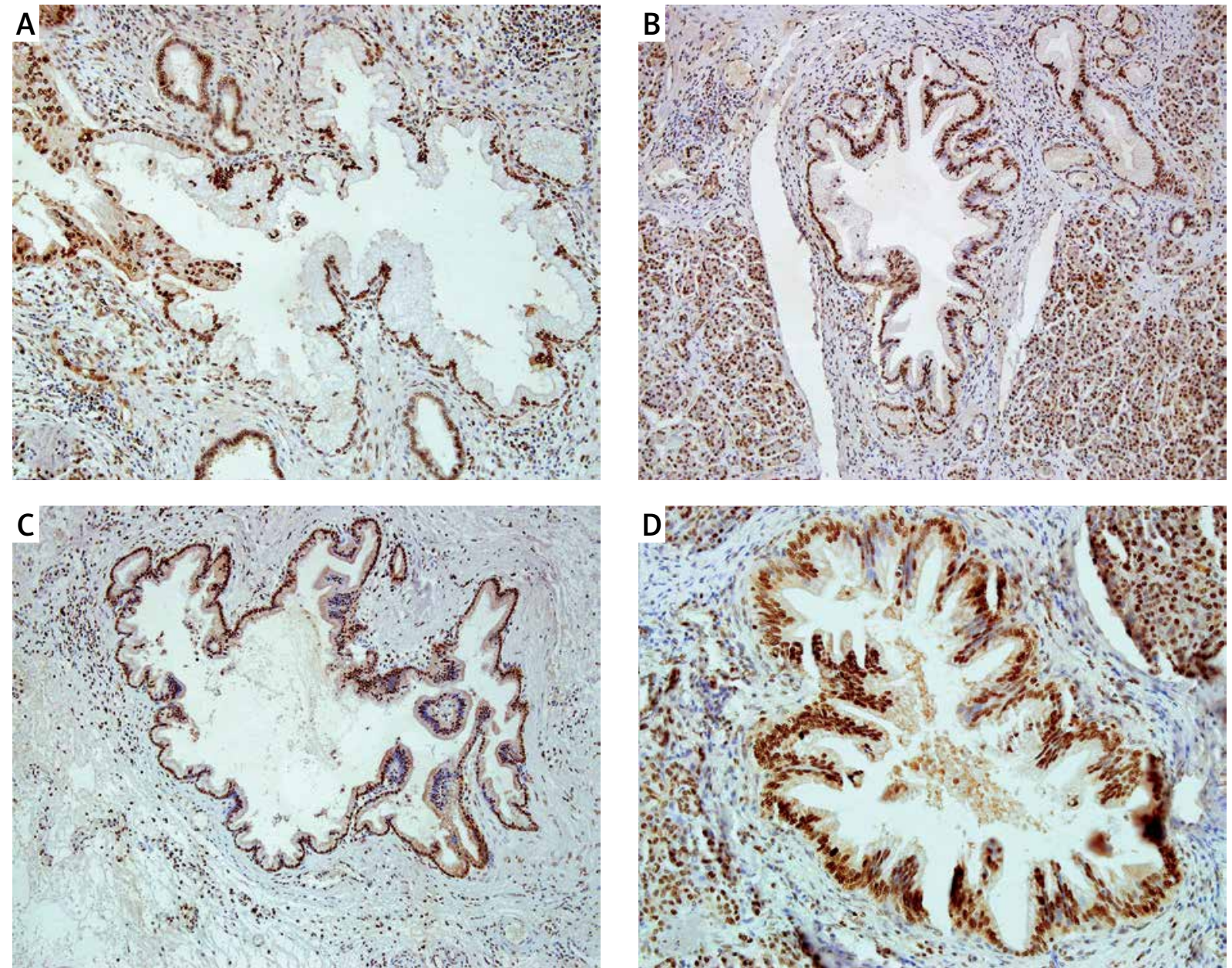

Figure 3. Nuclear immunoreactivity of PCNA in PanIN 1A (A), in PanIN 1B (B), in PanIN 2 (C), and in PanIN 3 (D).

Typical examples are shown

lial neoplasia. In normal ducts the mean expression of Ki67 was $1.2 \%, 3.1 \%$, in PanIN 1A, 5.0\% in PanIN 1B, $7.5 \%$, in PanIN 2, and $14.2 \%$ in PanIN 3. Karamitopoulou et al. [10] also assessed Ki67 expression in pancreatic intraepithelial neoplasia. In the normal ducts, this protein expression ranged from $1.0 \pm 0.0 \%, 2.1 \pm 0.5 \%$ in PanIN 3, while in invasive cancer the values were 6.7 $\pm 5.6 \%$. Ki67 expression differed between the normal ducts, PanIN 3, and pancreatic cancer. The author established that Ki67index was associated with a shorter survival time and may be an independent prognostic factor in patients with pancreatic cancer. Klein et al. [11] obtained the results similar to our study. Ki67 index equalled $0.41 \%$ in normal ducts, $0.69 \%$ in PanIN 1A, 2.33\% in PanIN 1B, 14.08\% in PanIN 2, and $22.01 \%$. in PanIN 3. This author determined also the Ki67 index in ductal pancreatic adenocarcinoma, which totalled $36.99 \%$. The differences in the expression of proteins were statistically significant between these groups. According to Klein, staging of dysplasia in the pancreatic ducts reflects proliferation undergoing in the epithelial cells of these ducts. Ki67 expression increases together with advancing staging of intraepithelial neoplasia in pancreas, which means intensified proliferation within the epithelium of pancreatic ducts. PCNA, a nuclear antigen of cellular proliferation, is another protein whose expression is caused by intense cell proliferation. PCNA is a helping protein for DNA polymerase $\delta$, which takes part in DNA synthesis and participates in cellular proliferation and regulation of the cell cycle via interactions with cyclins, cyclin-dependent kinases, and p21 protein [8]. PCNA overexpression was observed in many cancers of the alimentary canal, among others in colon cancer, stomach cancer, liver cancer, and pancreas cancer or in cancer of the colon, stomach, liver, and pancreas [12]. So far, this protein expression has not been examined in the development of precursor lesions of pancreatic cancer. In our study, the mean expression of PCNA was: $88.9 \%$ in normal ducts, $93.8 \%$ in pancreatic intraepithelial neoplasia of $1 \mathrm{~A}$ type, $93.7 \%$ 
Table III. Correlations between Ki67, cyclin D1, and PCNA expression and chosen clinicopathological parameters

\begin{tabular}{|c|c|c|c|c|c|c|}
\hline Variables & $\begin{array}{l}\text { Medium cyclin D1 } \\
\text { expression (\%) }\end{array}$ & $P$-value & $\begin{array}{l}\text { Medium Ki67 } \\
\text { expression (\%) }\end{array}$ & $P$-value & $\begin{array}{l}\text { Medium PCNA } \\
\text { expression (\%) }\end{array}$ & $P$-value \\
\hline Age: & & $<0.001$ & & $<0.001$ & & $<0.001$ \\
\hline$\leq 60$ & 13.4 & & 3.2 & & 91.7 & \\
\hline$>60$ & 25.6 & & 7.0 & & 95.9 & \\
\hline Gender: & & 0.725 & & $<0.001$ & & $<0.001$ \\
\hline Male & 20.6 & & 7.0 & & 96.1 & \\
\hline Female & 19.5 & & 3.6 & & 92.0 & \\
\hline Location: & & 0.075 & & 0.135 & & 0.591 \\
\hline Head & 22.4 & & 4.9 & & 94.6 & \\
\hline Corpus & 31.5 & & 5.8 & & 93.5 & \\
\hline Tail & 12.9 & & 3.3 & & 92.3 & \\
\hline Corpus and tail & 20.0 & & 8.9 & & 95.0 & \\
\hline Diagnosis: & & 0.923 & & 0.186 & & 0.031 \\
\hline Pancreatitis & 14.1 & & 2.4 & & 90.4 & \\
\hline Pancreatic ductal adenocarcinoma & 26.2 & & 7.6 & & 96.5 & \\
\hline Pancreatic cysts & 9.8 & & 2.8 & & 92.5 & \\
\hline PanIN: & & $<0.001$ & & $<0.001$ & & $<0.001$ \\
\hline Normal pancreatic ducts: & 0.7 & & 1.2 & & 88.9 & \\
\hline $1 \mathrm{~A}$ & 6.5 & & 3.1 & & 93.8 & \\
\hline $1 \mathrm{~B}$ & 19.2 & & 5.0 & & 93.7 & \\
\hline 2 & 35.7 & & 7.5 & & 96.5 & \\
\hline 3 & 59.5 & & 14.2 & & 97.6 & \\
\hline
\end{tabular}

Table IV. Comparison of the mean expression of Ki67, cyclin D1, and PCNA between various degrees of PanIN

\begin{tabular}{|c|c|c|c|c|c|c|}
\hline Protein & $\begin{array}{l}\text { Cyclin D1 ( } \\
\text { in \% with }\end{array}$ & $\begin{array}{l}\text { xpression } \\
\text {-value) }\end{array}$ & $\begin{array}{l}\text { Ki67 (me } \\
\text { in \% witl }\end{array}$ & $\begin{array}{l}\text { ression } \\
\text {-value) }\end{array}$ & $\begin{array}{l}\text { PCNA (m } \\
\text { in \% witl }\end{array}$ & $\begin{array}{l}\text { oression } \\
\text {-value) }\end{array}$ \\
\hline Normal vs. & $0.7 \pm 2.5$ & & $1.2 \pm 1.9$ & & $88.9 \pm 12.3$ & \\
\hline PanIN 1 & $12.9 \pm 13.8$ & $<0.001$ & $4.0 \pm 6.2$ & $<0.001$ & $93.8 \pm 8.1$ & $<0.001$ \\
\hline PanIN 2 & $35.7 \pm 20.1$ & $<0.001$ & $7.5 \pm 8.0$ & $<0.001$ & $96.5 \pm 6.9$ & $<0.001$ \\
\hline PanIN 3 & $59.5 \pm 21.3$ & $<0.001$ & $14.2 \pm 12.9$ & $<0.001$ & $97.6 \pm 5.4$ & $<0.001$ \\
\hline PanIN 1 vs. & $12.9 \pm 13.8$ & & $4.0 \pm 6.2$ & & $93.8 \pm 8.1$ & \\
\hline PanIN 2 & $35.7 \pm 20.1$ & $<0.001$ & $7.5 \pm 8.0$ & $<0.001$ & $96.5 \pm 6.9$ & $<0.001$ \\
\hline PanIN 3 & $59.5 \pm 21.3$ & $<0.001$ & $14.2 \pm 12.9$ & $<0.001$ & $97.6 \pm 5.4$ & $<0.001$ \\
\hline PanIN 2 vs. & $35.7 \pm 20.1$ & & $7.5 \pm 8.0$ & & $96.5 \pm 6.9$ & \\
\hline PanIN 3 & $59.5 \pm 21.3$ & $<0.001$ & $14.2 \pm 12.9$ & $<0.001$ & $97.6 \pm 5.4$ & $<0.001$ \\
\hline
\end{tabular}

in PanIN 1B, 96.5\% in PanIN 2, and 97.6\% in PanIN 3. Similarly to Ki67 protein, expression of nuclear antigen of nuclear proliferation was proven to increase together with staging of pancreatic intraepithelial neoplasia and resulted from intensive cellular proliferation within the epithelium of pancreatic ducts. In Dang's study [12] describing PCNA expression in pancreatic cancer, an increase accompanying histological staging of cancer was revealed in the expression of this protein. This may suggest that a gradual increase in PCNA expression ac- 


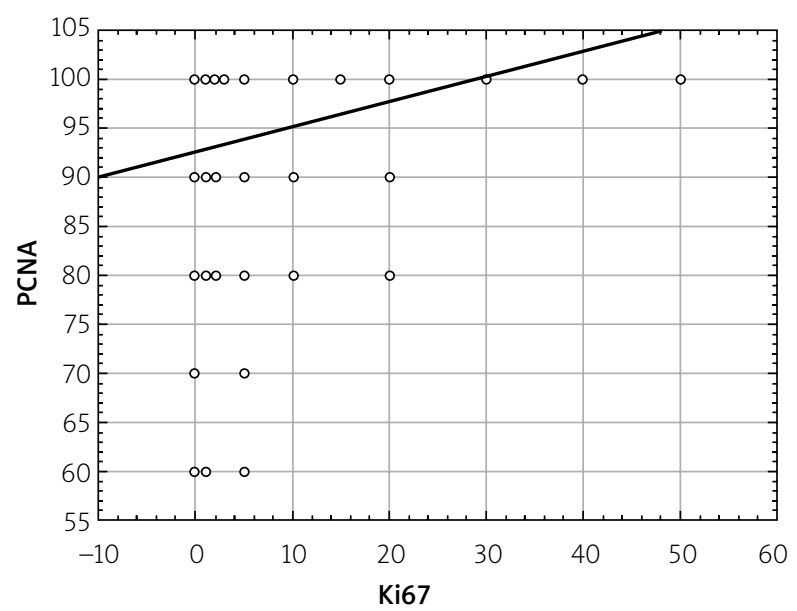

Figure 4. Correlations between total PCNA expression and total Ki67 expression (in the whole study group regardless of degree of PanIN)

companying growing staging of dysplasia in pancreatic ducts can also be observed in intraepithelial pancreatic neoplasia, which is a precursor lesion of cancer. Dang [12] revealed that a low expression of PCNA in patients with pancreatic cancer was related to longer survival time and could be a good prognostic agent. Moreover, he concluded that development of pancreatic cancer was caused by numerous genetic disorders among other mutations of suppressive genes responsible for limitation of cell proliferation and oncogenes, which lose the function of triggering mutator genes to repair the mistakes in DNA replication.

A negative regulator of the cell cycle for PCNA is cyclin D1, usually located in the cellular nucleus, from which it disappears during the S phase. Interaction of cyclin D1 with PCNA may prevent binding of a cellular proliferation antigen to a replication complex. This protein overexpression is a frequent phenomenon in the course of cancers and is usually caused by translocation of chromosomes, amplification of a gene, or excessive accumulation of protein [13]. Its overexpression was determined in breast cancer, oesophageal cancer, cancer of urinary bladder, head and neck, primary liver cancer, non-small-cell lung carcinoma, and coat cell lymphoma [14]. There are few findings describing the expression and significance of cyclin D1 in the course of pancreatic ductal adenocarcinoma and in precursor lesions. In our study, the mean expression of cyclin D1 was the following: $0.7 \%$ in normal ducts, $6.5 \%$ in PanIN 1A, 19.2\% in PanIN 1B, 35.7\% in PanIN 2, and $59.5 \%$ in PanIN 3. In the studies by Biankin et al. [15] overexpression of this protein was found in 4/21 (19\%) of PanIN cases. In a study by Al-Aynati et al. [16] overexpression of cyclin D1 was determined at the lev-

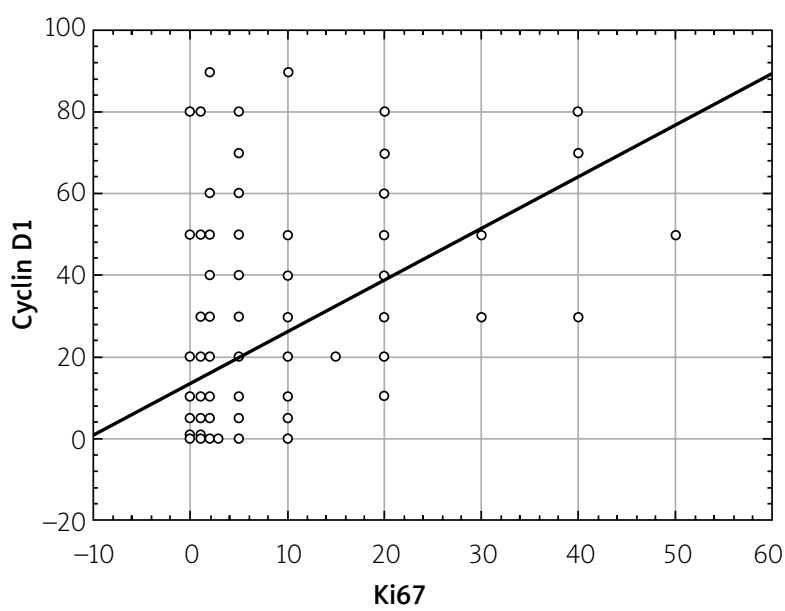

Figure 5. Correlations between total cyclin D1 expression and total Ki67 expression (in the whole study group regardless of degree of PanIN)

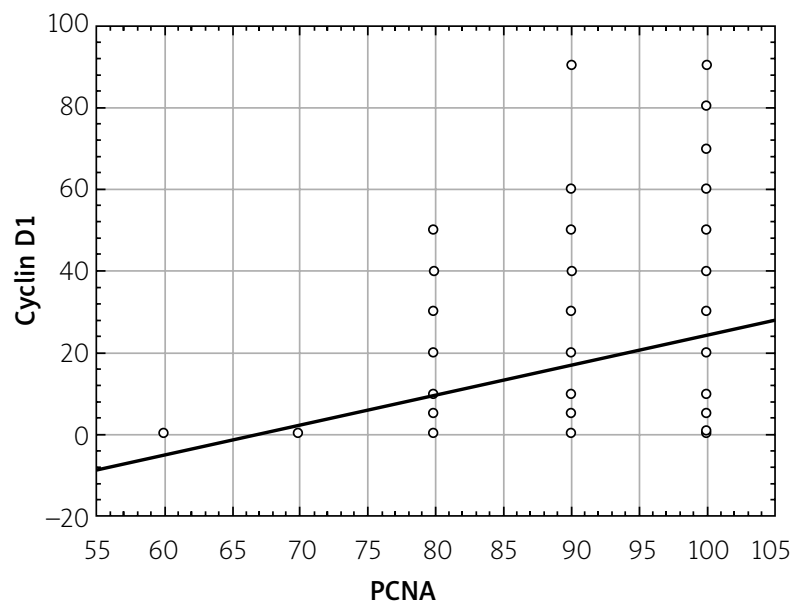

Figure 6. Correlations between total cyclin D1 expression and total PCNA expression (in the whole study group regardless of degree of PanIN)

el above $5 \%$ of positively staining cellular nuclei and was revealed in $22 \%$ of PanIN 2, $11 \%$ of PanIN 3, and $27 \%$ of pancreatic ductal adenocarcinoma cases. Both Biankin et al. [15] and Al-Aynati et al. [16] observed no positive expression of this protein in normal ducts as well as PanIN 1A and 1B. Additionally, they found that expression of D1 protein showed no correlation with PanIN staging. Al-Aynati [16] suggests that this protein played no significant role in intraepithelial neoplasia of pancreas. Maitra et al. [17] presented similar results in his study. The increased expression of D1 protein was observed in $29 \%$ of PanIN 2 cases and $57 \%$ of PanIN 3 cases, and its overexpression was regarded as a 'indirect' phenomenon in the multistage model of pancreatic cancer progression proceeding mutations of TP53 
gen and DPC4 inactivation. Overexpression of Cyclin D1 shortens the time of passing from G1 to S phase, promoting cellular progression and proliferation, which is one of the characteristics of neoplastic transformation. Evidence confirming the relation of this protein with carcinogenesis is the fact that cyclin is induced by activated myc and ras. oncogenes. According to Gansauge et al. [18], the presence of disorders in cyclin D1 expression is a relatively early phenomenon in the process of carcinogenesis because no differences were observed in this protein expression between cancers at the initial stage of development and the advanced stage. This theory can be confirmed in the examinations described in our study, in which a gradual increase in cyclin D1 expression reaching maximum values was found in lesions of advanced PanIN 3 (59.5\%).

Additionally, correlations between the expressions of these proteins were examined. Directly proportional correlations were proven between proteins: cyclin D1, Ki67, and PCNA, which means that an increase in the expression of one protein caused an increase in the expression of the other. No data describing the correlations between the proteins cyclin D1, Ki67, and PCNA were found in other authors' studies.

\section{Conclusions}

Expression of proteins Ki67, cyclin D1, and PCNA increased together with growing staging of pancreatic intraepithelial neoplasia, which indicates that these proteins may play a significant role in proliferation, which always accompanies cancerous transformation. Positive expression of these proteins present in the pancreatic ducts with pancreatic intraepithelial neoplasia of low staging - PanIN 1, moderate staging - PanIN 2, and advanced staging - PanIN 3 emphasises the fact that uncontrolled cellular multiplication takes place not only in cancer of advanced staging but also in precursor lesions. Overexpression of proteins Ki67, cyclin D1, and PCNA may be helpful in differential diagnostics of individual advancement stages of pancreatic intraepithelial neoplasia.

\section{Conflict of interest}

The authors declare no conflict of interest.

\section{References}

1. Siegel RL, Miller KD, Jemal A. Cancer Statistics. CA Cancer J Clin 2017; 67: 7-30.

2. Słotwiński R, Słotwińska SM. Diagnostic value of selected markers and apoptotic pathways for pancreatic cancer. Cent Eur J Immunol 2016; 41: 392-403.
3. Andea AA, Basturk O, Adsay NV. Pancreatic intra-epithelial neoplasia: current clinicopathological and molecular considerations. Curr Diagn Pathol 2005; 11: 80-94.

4. Scholzen T, Gerdes J. The Ki67 protein: from the known and the unknown. J Cell Physiol 2000; 182: 311-22.

5. Brown DC, Gatter KC. Ki67 protein: the immaculate deception? Histopathology 2002; 40: 2-11.

6. Alao JP. The regulation of cyclin D1 degradation: roles in cancer development and the potential for therapeutic invention. Mol Cancer 2007; 6: 24.

7. Naryzhny SN. Proliferating cell nuclear antigen: a proteomics view. Cell Mol Life Sci 2008; 65: 3789-808.

8. Wang SC. PCNA: a silent housekeeper or a potential therapeutic target? Trends Pharmacol Sci 2014; 35: 178-86.

9. Linder S, Parrado C, Falkmer UG, et al. Prognostic significance of Ki67 antigen and p53 protein expression in pancreatic duct carcinoma: a study of the monoclonal antibodies MIB-1 and DO07 in formalin-fixed parafin-embedded tumour material. $\mathrm{Br}$ J Cancer 1997; 76: 54-9.

10. Karamitopoulou E, Zlobec I, Tornillo L, et al. Differential cell cycle and proliferation marker expression in ductal pancreatic adenocarcinoma and pancreatic intraepithelial neoplasia (PanIN). Pathology 2010; 42: 229-34.

11. Klein WM, Hruban R, Klein-Szanto AJ, et al. Direct correlation between proliferative activity and dysplasia in pancreatic intraepithelial neoplasia (PanIN): additional evidence for a recently proposed model of progression. Mod Pathol 2002; 15: 441-7.

12. Maga G, Hubscher U. Proliferating cell nuclear antigen (PCNA): a dancer with many partners. J Cell Sci 2003; 116: 3051-60.

13. Dang CX, Han Y, Qin ZY, et al. Clinical significance of expression of $\mathrm{p} 21$ and $\mathrm{p} 53$ proteins and proliferating cell nuclear antigen in pancreatic cancer. Hepatobiliary Pancreat Dis Int 2002; 1: 302-5.

14. Yang K, Hitomi M, Stacey DW. Variations in cyclin D1 levels through the cell cycle determine the proliferative fate of a cell. Cell Div 2006; 1: 32.

15. Biankin AV, Kench JG, Biankin SA. Pancreatic intraepithelial neoplasia in association with intraductal papillary mucinous neoplasms of the pancreas: implications for disease progression and recurrence. Am J Surg Pathol 2004; 28: 1184-92.

16. Al-Aynati MM, Radulovich N, Ho J, et al. Overexpression of G1-S cyclins and cyclin-dependent kinases during multistage human pancreatic duct cell carcinogenesis. Clin Cancer Res 2004; 10: 6598-605.

17. Maitra A, Adsay NV, Argani P, et al. Multicomponent analysis of the pancreatic adenocarcinoma progression model using a pancreatic intraepithelial neoplasia tissue microarray. Mod Pathol 2003; 16: 902-12.

18. Gansauge S, Gansauge F, Ramadani M, et al. Overexpression of cyclin D1 in human pancreatic carcinoma is associated with poor prognosis. Cancer Res 1997; 57: 1634-7.

Received: 21.06 .2017

Accepted: 15.11.2017 